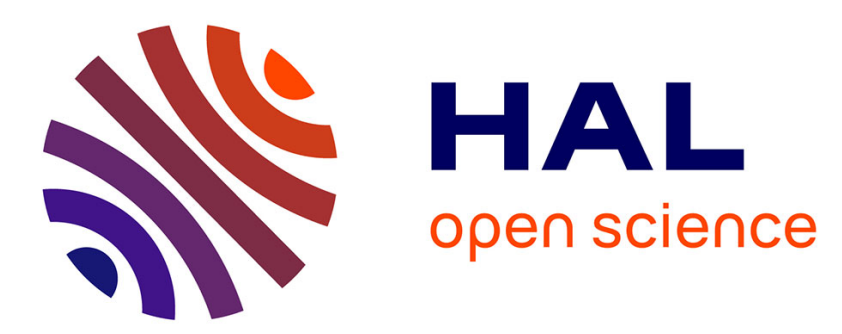

\title{
Une science de la rencontre. L'écologie scientifique au service de formations interdisciplinaires
}

Hélène Barbé, Caroline Vincent, Cécile Blatrix, Nathalie Frascaria-Lacoste

\section{To cite this version:}

Hélène Barbé, Caroline Vincent, Cécile Blatrix, Nathalie Frascaria-Lacoste. Une science de la rencontre. L'écologie scientifique au service de formations interdisciplinaires. Annales des mines - Série Responsabilité et environnement, 2021, 2021/1 (101), pp.37-40. 10.3917/re1.101.0037 . hal-03137827

\section{HAL Id: hal-03137827 https://hal.science/hal-03137827}

Submitted on 12 Nov 2021

HAL is a multi-disciplinary open access archive for the deposit and dissemination of scientific research documents, whether they are published or not. The documents may come from teaching and research institutions in France or abroad, or from public or private research centers.
L'archive ouverte pluridisciplinaire HAL, est destinée au dépôt et à la diffusion de documents scientifiques de niveau recherche, publiés ou non, émanant des établissements d'enseignement et de recherche français ou étrangers, des laboratoires publics ou privés. 


\title{
Une science de la rencontre \\ L'écologie scientifique au service de formations interdisciplinaires
}

\author{
Par Hélène BARBÉ ${ }^{1}$ \\ Laboratoire Écologie, systématique et évolution (ESE) de l'Université Paris-Saclay \\ Caroline VINCENT ${ }^{1}$ \\ Laboratoire ESE de l'Université Paris-Saclay \\ Cécile BLATRIX \\ Professeure de science politique à AgroParisTech \\ Laboratoire PRINTEMPS - Professions, Institutions, Temporalités (CNRS-UVSQ) \\ et Nathalie FRASCARIA-LACOSTE \\ Professeure d'Écologie évolutive et ingénierie écologique à AgroParisTech \\ Laboratoire ESE de l'Université Paris-Saclay
}

Dans cet article, nous revenons sur ce qui constitue le cœur de l'écologie scientifique, et montrons comment cette discipline peut enrichir les formations interdisciplinaires à plusieurs égards. L'écologie a longtemps eu tendance à laisser l'être humain en dehors de son champ d'étude. Or, au regard des crises que nous vivons actuellement, la perspective écologique a pris un tournant pour s'orienter vers des approches plus transversales. Nous verrons comment cette science de la rencontre (rencontre des disciplines, des courants de pensées, des valeurs, des rationalités...) et de la connaissance du sensible permet un changement de paradigme au travers de concepts clés, tels que les (socio)-écosystèmes, la complexité, la dynamique, les interrelations ou encore les incertitudes. Les questionnements et savoirs issus de l'écologie scientifique ont donc vocation à favoriser une meilleure compréhension générale des fonctionnements des sociétés humaines imbriquées au sein des systèmes vivants.

\section{Introduction}

L'écologie est une science jeune. Née sous la plume de Haeckel qui la définit, en 1866, comme la science des relations des organismes avec le monde environnant, elle s'est construite comme une nouvelle discipline scientifique dès la fin du XIXème siècle dans un cadre culturel de type anglo-saxon. Cette discipline s'est longtemps focalisée essentiellement sur le monde vivant non humain en étudiant les dynamiques naturelles et leurs évolutions multiples. L'écologie a grandi dans un cadre interdisciplinaire choisi ne s'intéressant que très peu aux humains, sauf pris comme des éléments perturbateurs des écosystèmes. La profonde crise de la biodiversité que nous vivons aujourd'hui invite à une autre interdisciplinarité. Les sciences sociales, en particulier, ont trop peu été mobilisées par l'écologie. Elles permettent pourtant l'accès à des grilles de lecture essentielles faisant le lien entre la

\footnotetext{
${ }^{1}$ Co-premières auteures.
} 
société et le monde vivant non humain, et apportent des éléments de réponse à ce que l'écologie pose elle-même dans ses constats et conclusions.

\section{De quelle science s'agit-il donc ?}

Comme nous la définissions précédemment, l'écologie est la science des relations des organismes avec le monde environnant, c'est-à-dire la science des conditions d'existence. L'écologie est née à l'intersection de la biologie des organismes et de plusieurs sciences physiques, dont la thermodynamique. Son champ disciplinaire comprend notamment les sous-disciplines que sont la génétique et la biologie des populations, l'évolution darwinienne, l'écophysiologie ou l'écologie du paysage.

En étudiant des objets définis à différentes échelles spatiales et selon différentes temporalités, l'écologie côtoie aussi indubitablement les autres sciences, comme la physique, les mathématiques, la science politique, la sociologie, ou encore la psychologie et la philosophie, pour ne citer qu'elles. En tant que discipline relativement récente - malgré ses relations avec les sciences physiques et biologiques, elle a fait l'objet d'un processus d'autonomisation et d'institutionnalisation progressif. Les «écologues » (terme datant des années 1980) ont dû se démarquer des « écologistes » afin d'affirmer l'écologie comme une science à part entière, et l'écologie est encore confrontée à des enjeux de reconnaissance au sein du champ scientifique où l'idée d'assujettir la nature reste bien présente ${ }^{2}$. C'est bien parce que la question écologique ne peut être réduite à des problématiques ne relevant que des « sciences dures » qu'elle est fondamentalement si pluri-, trans-, poly- et interdisciplinaire. Par ailleurs, l'écologie est un « modèle d'une science qui définit très peu et qui évite les généralités, une science de savoirs qui se cultivent » (Stengers, 2019).

De fait, l'écologie est une science de la rencontre : de la rencontre des disciplines et des courants de pensées, mais également des différentes rationalités ${ }^{3}$. Elle invite en effet à articuler les différents types de rationalité distingués par Max Weber, sans tomber dans un usage mécanique de sa conceptualisation qui peut conduire à ne considérer comme « rationnelle » que la «pure rationalité téléologique ». La typologie proposée par Weber, finement analysée et discutée par Catherine ColliotThélène, distingue quatre déterminants de l'action sociale qui peuvent s'appliquer aux enjeux écologiques : la rationalité pragmatique, qui vise à atteindre des fins réfléchies, telles que la démonstration de phénomènes écologiques ; la rationalité " en valeur », qui traduit l'engagement d'un individu ou d'un groupe d'individus selon des valeurs ou des impératifs éthiques ou religieux, comme, dans la lutte pour la protection de la biodiversité, l'idée selon laquelle « plus est mieux » ${ }^{4}$; la rationalité qualifiée d'instinctive, d'immédiate, liée à l'expression des affects et qui peut se traduire par le besoin de protection en réponse à l'ensemble des affects suscités par

\footnotetext{
${ }^{2}$ Voir, par exemple, le rapport de mandature 2010 - 2014 du Conseil scientifique de l'Institut écologieenvironnement du CNRS, faisant état d'une «condescendance » de la part des sciences dites « dures », qui s'expliquerait par un manque de culture scientifique en écologie (INEE, 2014, p. 25).

${ }^{3}$ Rationnel pragmatique, rationnel en valeur, rationnel selon les affects ou des émotions, et enfin, rationnel traditionnel.

${ }^{4}$ Idée qui fait, à ce jour, toujours débat entre les chercheurs écologues (Sutherland et al., 2013).
} 
les objets de l'écologie, tels que des paysages, les êtres vivants, etc. ; et enfin, la rationalité « traditionnelle », combinant rationalité des affects et cadre culturel sociologique, qui peut se traduire par les moyens de qualification des espèces « invasives » ou « en danger », etc. C'est en intégrant dans l'analyse l'ensemble de ces rationalités, et non en stigmatisant l'irrationalité supposée de certains acteurs sociaux, que l'écologie peut permettre d'éclairer la décision et l'action.

Ainsi, l'écologie scientifique est intimement liée à la connaissance du sensible, de ce que les écologues perçoivent, mais également à ce qu'ils ressentent et apprécient, ainsi qu'aux cadres socio-culturels dans lesquels ils évoluent. Comme toute science, mais de façon peut-être plus frappante, elle ne peut s'abstraire de tout jugement de valeur sur les objets de ses études, puisque ceux-ci déterminent, au moins en partie, leurs modalités d'appréhension. C'est pourquoi est si important le fait que l'écologie scientifique agisse en réseau, combine les savoirs et les techniques entre les différentes disciplines : la multiplication et la diversification des réalités interprétées par ses chercheurs lui confèrent alors le statut de science « la plus humaine des sciences de la nature » (Deléage, 1992).

\section{Une science en transition}

L'écologie scientifique nous permet également d'entrevoir un changement de paradigme en ce qu'elle favorise le passage de la notion de « système », qui est défendue, entre autres, par la physique et l'ingénierie - avec les idées bien connues que sont le rendement, la constance, la prédictibilité, la stabilité autour d'un équilibre (résilience ingénieuriale) et le maintien du rendement d'une fonction (optimisation) à la notion d' « écosystème », qui, elle, va porter les notions de persistance, de durabilité, de changement, d'imprédictibilité, de conditions toujours loin des équilibres ou d'instabilités (résilience écologique).

L'écosystème peut être défini au sens large comme : « L'ensemble du système..., y compris non seulement le complexe d'organismes, mais aussi tout le complexe des facteurs physiques formant ce que nous appelons l'environnement » (Tansley, 1935). Le plus souvent, les études dédiées aux interactions humains-milieux ou aux écosystèmes positionnent l'humain en dehors du système naturel, ce qui, de fait, limite la capacité à prendre en considération la complexité des interactions existantes entre l'être humain et la nature (Redman et al., 2004 ; Liu et al., 2007 ; Folke, 2007). C'est pourquoi, depuis quelques temps maintenant, les écologues explorent de plus en plus leurs liens avec les sciences humaines, une relation dont les racines sont profondes (Pickett et Cadenasso, 2002). Effectivement, l'écologie va opérer un changement disciplinaire conceptuel et s'orienter vers l'analyse des jeux d'acteurs dans les écosystèmes. Dans la lignée des travaux d'Elinor Ostrom (Ostrom, 2009), l'écologie scientifique va se saisir de plus en plus de la notion de socio-écosystème (SE), définie comme « un système interactif entre deux systèmes constitués par un (ou des) socioécosystème(s) et par un (ou des) écosystème(s) naturel(s) et/ou artificialisé(s) s'inscrivant dans un espace géographi[qu]e donné et évoluant dans le temps » (Morin, 2018).

Ainsi, l'objet scientifique de la discipline évolue et se tourne vers l'étude des interactions entre la dynamique des systèmes naturels et artificialisés et les sociétés 
humaines (ainsi, les milieux naturels restent des ressources, mais deviennent aussi, de fait, un cadre de relations sociales). La compréhension de ces SE et leur explicitation dans un enseignement interdisciplinaire revêtent un caractère qui dépasse la notion elle-même : le constat fait que le fonctionnement des écosystèmes se rapproche également de ceux de l'humain, permet ainsi une meilleure compréhension générale des fonctionnements des sociétés humaines.

\section{Enseigner l'incertitude}

À l'image des systèmes vivants composant les SE qui sont dynamiques et évolutifs, il devient essentiel pour les chercheurs de se placer dans une dynamique permanente de réflexion critique de la connaissance acquise tant les processus qui la caractérisent sont multiscalaires, multi-temporels et constamment en évolution. L'écologie nous pousse donc vers l'acceptation du changement, l'imprédictibilité ou l'absence d'équilibres fixes et permanents, qui sont gages de durabilité.

Edgar Morin disait «On peut parler simplement de la complexité. ». Comme il l'explique, "les sciences nous ont fait acquérir beaucoup de certitudes, mais nous ont également révélé au cours du $X X^{e}$ siècle d'innombrables domaines d'incertitude » (Morin, 2006). Aussi, il considère que l'enseignement des incertitudes apparues (entre autres) dans les sciences physiques, biologiques et historiques est indispensable, ce que traduit l'idée suivante : " Il faut apprendre à naviguer dans un océan d'incertitudes à travers des archipels de certitude. » (Morin, 2006). Dans l'écologie scientifique, avec l'introduction des SE, les incertitudes et la complexité sont omniprésentes, notamment au travers de ce que l'on appelle «l'autoorganisation » de tous les systèmes vivants et du caractère spontané de celle-ci, que certains auteurs qualifient à la fois de processus et de système (Moreno, 2004). La question de l'incertitude est également devenue centrale en sciences sociales, comme le montre bien, par exemple, l'analyse des controverses socio-techniques (Callon et al., 2001).

Dans la continuité, c'est en réaction à l'autopoïèse - que l'on peut définir comme la capacité des systèmes vivants à “ s'auto-organiser " ${ }^{5}$, c'est-à-dire que «le système produit des effets résultant de son activité, mais, circulairement, ces effets maintiennent le système qui les cause » (Mossio et Bich, 2014) - qu'lsabelle Stengers (2019) invoque le concept de sympoïèse, tel que proposé par Donna Haraway : "Cette co-création permanente du vivant, dont parle Donna Haraway, signifie simplement faire avec, ou faire grâce aux autres et au risque des autres. Les vivants sont tous actifs, ils font ; mais ce qu'ils font implique, présuppose ou crée des rapports les uns avec les autres. Ils sont impliqués les uns avec les autres. Et, ensemble, ils font des mondes ». Cette idée de sympoïèse souligne l'importance de l'interdépendance entre les systèmes vivants tous confondus et renvoie à notre propre condition. Intégrer l'écologie dans nos formations, c'est ramener au cœur de nos préoccupations le respect de l'autre et l'humilité au regard de notre présence éphémère, et c'est remettre en question nos rapports au vivant et au non-vivant dans le but d'imaginer non pas un monde « certain » où l'humain contrôle l'ensemble des

\footnotetext{
${ }^{5}$ Terme utilisé par Emmanuel Kant dans Critique de la faculté de juger [1790], Paris, Vrin, 1993.
} 
paramètres, mais un monde « incertain » (Callon et al., 2001) dans lequel l'existence même de l'humain est questionnée.

Enfin, il nous semble important de bien saisir la teneur de cette « vocation » interdisciplinaire. Elle renvoie en effet à la question des différents savoirs mobilisés, notamment des savoirs dits « scientifiques ». Leur confrontation aux savoirs « non scientifiques » - que d'autres appellent « empiriques », «locaux » ou « profanes »permettrait d'élargir la portée de l'écologie, en sachant que ces deux types de savoirs sont forcément soumis à « l'influence d'une culture et d'une histoire locales » (Brun et al., 2007). Or, ce que nous apprennent la pratique et la pensée en écologie, c'est que l'humain est plus que jamais face à des problèmes complexes et multi-acteurs sur lesquels sa «maîtrise » reste très partielle. Par conséquent, plus les problèmes, situations, enjeux se complexifient, et plus les acteurs doivent être « familiarisé[s] avec la pratique interdisciplinaire » (Brun et al., 2007), et ce non seulement du point de vue des connaissances et des faits, mais aussi du point de vue des valeurs. En cela, l'éthique écologique nous ramène au principe de responsabilité de l'espèce humaine, responsabilité qui se manifeste dans nos choix individuels, collectifs et communs. Ainsi, le choix d'imprégner chaque étape et chaque champ de nos formations par les savoirs, mais surtout par les questions et les incertitudes que porte l'écologie, nous paraît plus que jamais nécessaire, dans une perspective d'interpénétration des vécus, des trajectoires et des changements à opérer.

\section{Conclusion}

Comme nous venons de l'évoquer, l'écologie scientifique d'aujourd'hui est marquée par le passage à des approches multidisciplinaires et multiscalaires se combinant avec un changement de posture récent des écologues, mais aussi avec celui des objets de leurs études. Ce changement de posture fait que les chercheurs écologues travaillent maintenant de plus en plus à l'échelle des socio-écosystèmes. Si l'écologie scientifique, dans les formations dédiées, permettait déjà à des étudiants venant d'autres disciplines d'acquérir une meilleure compréhension du fonctionnement des systèmes vivants avec leurs logiques de dynamiques imprédictibles et évolutives, en introduisant l'humain comme un élément constituant de ce monde vivant au même titre que les autres éléments, elle va par l'interrogation autre qu'elle fait aujourd'hui de notre relation au monde trouver là une nouvelle légitimité essentielle dans un enseignement interdisciplinaire orienté vers des principes de durabilité forte.

\section{Bibliographie}

BRUN É., BETSCH J. M., BLANDIN P., HUMBERT G., LEFEUVRE J. C. \& MARINVAL M. C. (2007), «Postures des scientifiques et interdisciplinarité dans le champ de l'environnement », Natures Sciences Sociétés, n¹5, pp. 177-185. 
CALLON M., LASCOUMES P. \& BARTHE Y. (2001), Agir dans un monde incertain. Essai sur la démocratie technique, Paris, Le Seuil, coll. "La couleur des idées », $358 \mathrm{p}$.

COLLIOT-THÉLÈNE C. (2011), « Retour sur les rationalités chez Max Weber », Les Champs de Mars, $n^{\circ} 22$, pp. 13-30.

DELÉAGE J. P. (1992), « Documentation : aux origines de la science écologique, à propos de quelques ouvrages récents », Revue Histoire des Sciences, pp. 477-490.

DEVICTOR V., CARRIERE S. \& GUILLET F. (2016), « Protection de la nature et valeurs », dans ROCHE P., GEIJZENDORFFER I., LEVREL H. \& MARIS V. (Eds.), Valeurs de la biodiversité et services écosystémiques. Perspectives interdisciplinaires, Versailles, Éditions Quæ, coll. « Update Sciences \& Technologies », pp. 39-54.

DUPUY M. (2004), Les cheminements de l'écologie en Europe : une histoire de la diffusion de l'écologie au miroir de la forêt 1880 - 1980, L'Harmattan, coll. « Biologie, Ecologie, Agronomie », $297 \mathrm{p}$.

FOLKE C. (2007), "Social-ecological systems and adaptive governance of the commons", Ecological Research, 22, pp. 14-15.

INSTITUT ÉCOLOGIE-ENVIRONNEMENT DU CNRS (2014), Rapport de mandature du Conseil scientifique de l'INEE, 2010-2014, sous la coordination de Jean-Denis Vigne, $58 \mathrm{p}$.

KANT E. (1993), Critique de la faculté de juger, [1790] Paris, Vrin, 380 p.

LIU J., DIETZ T., CARPENTER S. R., ALBERTI M., FOLKE C., MORAN E., PELL A. N., DEADMAN P., KRATZ T., LUBCHENCO J., OSTROM E., OUYANG Z., PROVENCHER W., REDMAN C. L., SCHNEIDER S. H. \& TAYLOR W. W. (2007), "Complexity of coupled human and natural systems", Science, 317, 5844, pp. 15131516.

REDMAN C. L., GROVE J. M. \& KUBY L. H. (2004), "Integrating social science into the Long-Term Ecological Research (LTER) network: Social dimensions of ecological change and ecological dimensions of social change", Ecosystems, 7, pp. 161-171.

MORENO A. (2004), « Auto-organisation, autonomie et identité », Revue internationale de philosophie, n²28, pp. 135-150.

MORIN E. (2006), «Les sept savoirs nécessaires », Revue du MAUSS, n²8, pp. 5969.

MORIN O. (2018), «La formation de la pensée écologique, éducation scientifique et politique. ", dans PERRU O., LAUTESSE P. \& CHARLES F. (Eds.), Une éducation à la pensée scientifique pour une société plus juste, Paris, Vrin, pp. 125-146. 
MOSSIO M. \& BICH L. (2014), " La circularité biologique : concepts et modèles », dans VARENNE F. et al. (Eds.), Modéliser et simuler. Épistémologies et pratiques de la modélisation et de la simulation, tome 2, Paris, Éditions Matériologiques, coll. «Modélisations, simulations, systèmes complexes », pp. 137-170.

OSTROM E. (2009), "A general framework for analyzing sustainability of socioecological systems", Science, 325, pp. 419-422.

PICKETT S. T. A. \& CADENASSO M. L. (2002), "The ecosystem as a multidimensional concept: Meaning, model, and metaphor", Ecosystems, 5(1), pp. 110.

STENGERS I. (2019), Résister au désastre, Wildproject Editions, coll. « Dialogue », $87 \mathrm{p}$.

SUTHERLAND W. J., FRECKLETON R. P., GODFRAY H. C. J., BEISSINGER S. R., BENTON T., CAMERON D. D., CARMEL Y., COOMES D. A., COULSON T., EMMERSON M. C. et al. (2013), "Identification of 100 fundamental ecological questions." Journal of Ecology, 101, pp. 58-67.

TANSLEY A. G. (1935), "The use and abuse of vegetational terms and concepts" Ecology, 16(3), pp. 284-307. 